\title{
Giant cell tumor of the patella: An uncommon cause of anterior knee pain
}

\author{
TATSUYA SHIBATA $^{1}$, JUN NISHIO ${ }^{1}$, TAIKI MATSUNAGA ${ }^{1}$, MIKIKO AOKI $^{2}$, \\ HIROSHI IWASAKI $^{2}$ and MASATOSHI NAITO ${ }^{1}$
}

Departments of ${ }^{1}$ Orthopaedic Surgery and ${ }^{2}$ Pathology, Faculty of Medicine, Fukuoka University, Fukuoka 814-0180, Japan

Received July 11, 2014; Accepted July 21, 2014

DOI: $10.3892 / m c o .2014 .433$

\begin{abstract}
The patella is a rare site for the development of primary tumors. This is the case report of a giant cell tumor (GCT) occurring in the patella in a 25 -year-old woman. The patient presented with a 1-year history of occasional right anterior knee pain. The radiological characteristics suggested a benign condition. The intraoperative pathological diagnosis was GCT of the bone. The lesion was treated by radical curettage with adjuvant therapy comprising phenol and ethanol and injection of calcium phosphate cement. Histologically, the tumor consisted of round or spindle-shaped mononuclear cells admixed with numerous osteoclastic giant cells. The patient was asymptomatic and there was no evidence of local recurrence or distant metastasis 16 months after surgery. Although rare, patellar GCT may be included in the differential diagnosis of anterior knee pain and/or swelling, particularly in young adults.
\end{abstract}

\section{Introduction}

Primary patellar tumors are uncommon and may present with anterior knee pain and/or swelling (1). Recently, Casadei et al (2) reported that the most frequent benign patellar tumor is giant cell tumor (GCT), followed by chondroblastoma and aneurysmal bone cyst (ABC). GCT accounts for $4-5 \%$ of all primary bone tumors and usually affects the ends of long bones (3). This is the report of a rare case of GCT of the patella in a young adult woman and a brief review of the relevant literature. Written informed consent was obtained from the patient for publication of this case report and accompanying images.

Correspondence to: Dr Jun Nishio, Department of Orthopaedic Surgery, Faculty of Medicine, Fukuoka University, 7-45-1 Nanakuma, Jonan-ku, Fukuoka 814-0180, Japan

E-mail:jnishio@cis.fukuoka-u.ac.jp

Key words: anterior knee pain, giant cell tumor, imaging, patella

\section{Case report}

A 25-year-old woman presented with a 1-year history of occasional right anterior knee pain. Two weeks prior to visiting our hospital, the patient had been experiencing increasing anterior knee pain following a stumble. The physical examination revealed mild swelling and slight tenderness in the anterior aspect of the right knee. However, we did not observe any skin adhesion, local heat, redness, or limitation of the knee joint range of motion. Furthermore, no joint effusion was noted. The laboratory data were within normal limits, except for a marginal elevation of alkaline phosphatase levels. The patient's past medical history was unremarkable.

Plain radiographs revealed a well-defined, lytic lesion in the inferior and central portion of the patella (Fig. 1). There was no periosteal reaction. Computed tomographic (CT) scans demonstrated an intraosseous lytic lesion with associated cortical thinning (Fig. 2). On magnetic resonance imaging (MRI), the lesion exhibited slightly higher signal intensity compared to skeletal muscle on T1-weighted sequences (Fig. 3A) and heterogeneous signal intensity on T2-weighted spectral presaturation with inversion recovery sequences (Fig. 3B). Contrast-enhanced fat-suppressed T1-weighted sequences demonstrated a heterogeneously strong enhancement of the lesion (Fig. 3C). There was no obvious soft tissue or intra-articular extension. Technetium-99m hydroxymethylene diphosphonate bone scintigraphy revealed no abnormal uptake, except for the right patella (Fig. 4). Based on these findings, a benign bone tumor was suggested, including GCT in the differential diagnosis.

An incisional biopsy was performed and a rapid intraoperative pathological diagnosis was GCT of the bone. The patient subsequently underwent radical intralesional curettage with a high-speed burr. Following curettage, $70 \%$ phenol was applied meticulously to the inner surface of the cavity with a cotton swab for a period of $5 \mathrm{~min}$. The cavity was then filled with 99.5\% ethanol for $5 \mathrm{~min}$. After extensive washing with normal saline solution, the cavity was filled with calcium phosphate cement (Biopex ${ }^{\circledR}$; Pentax, Tokyo, Japan). Microscopically, the tumor consisted of round or spindle-shaped mononuclear cells admixed with numerous osteoclastic giant cells (Fig. 5). Foci of hemorrhage, hemosiderin deposits and aggregates of foamy histiocytes were also found. Mitotic figures were present 

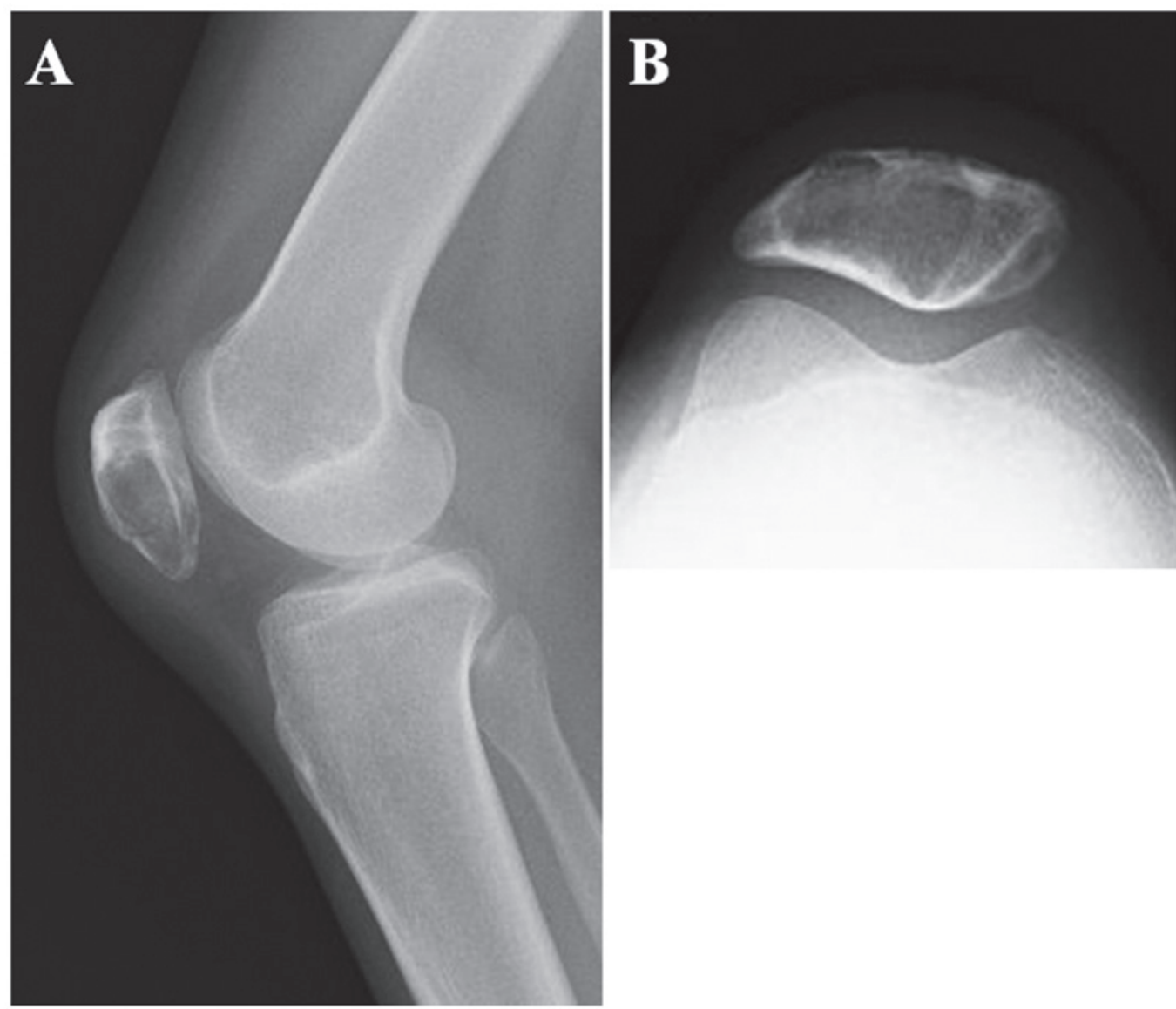

Figure 1. Preoperative (A) lateral and (B) axial radiographs of the right knee reveal a well-defined, lytic lesion in the inferior and central portion of the patella.
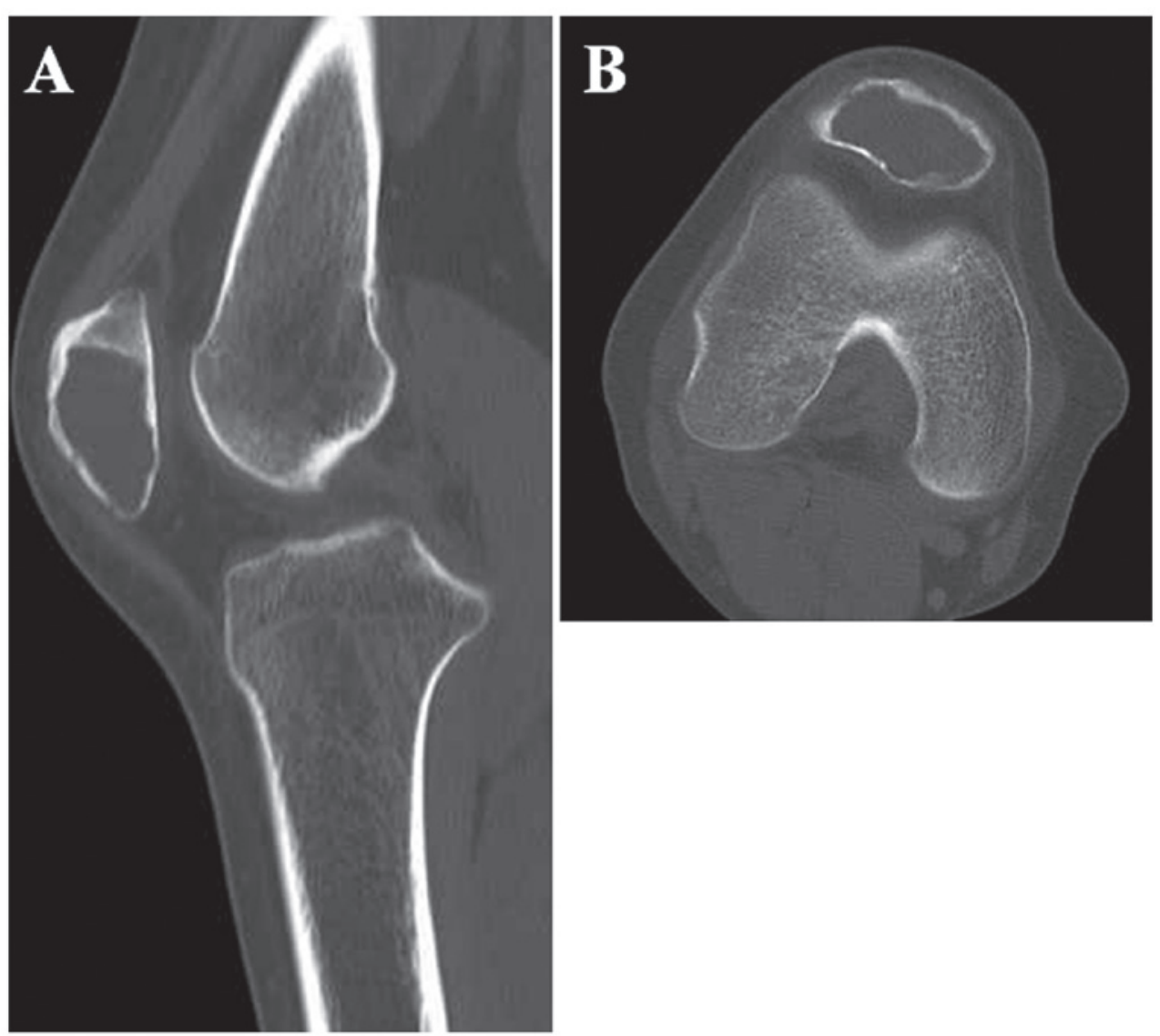

Figure 2. (A) Lateral and (B) axial computed tomographic scans of the right knee show an intraosseous lytic lesion with cortical thinning in the patella. 

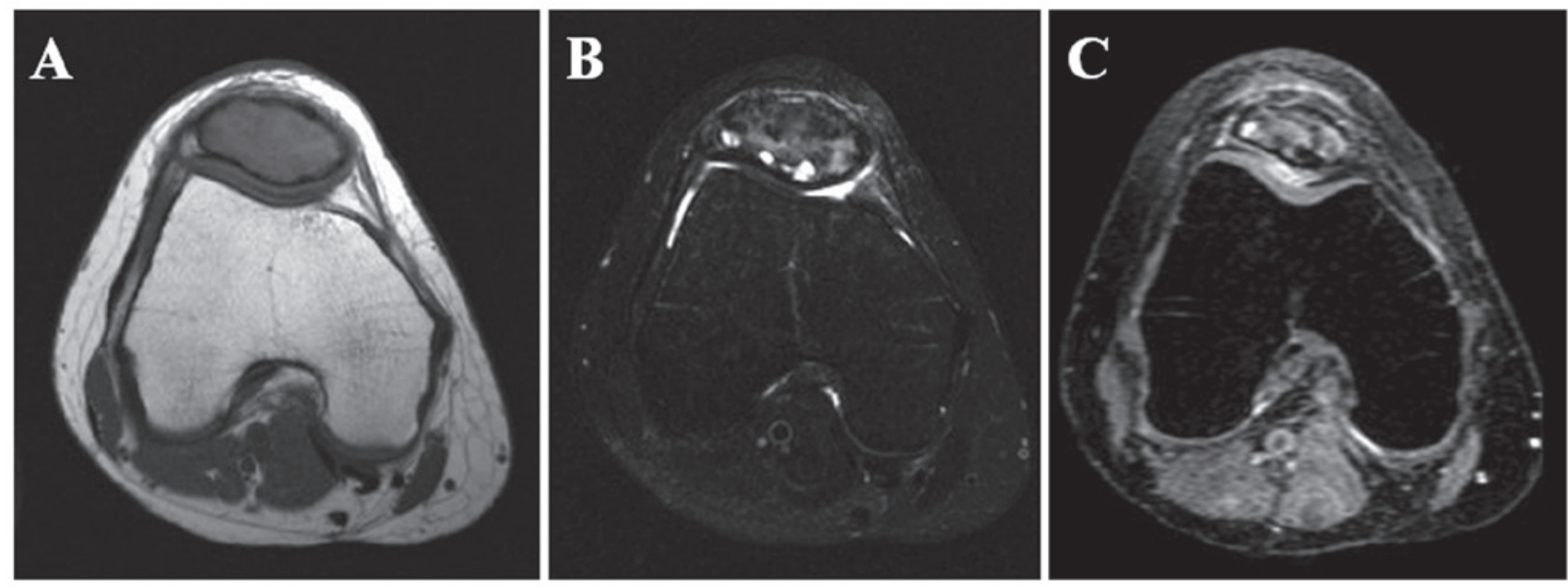

Figure 3. Axial magnetic resonance images of an intraosseous lesion in the right patella. (A) The lesion exhibits slightly higher signal intensity relative to skeletal muscle on T1-weighted sequence and (B) heterogeneous signal intensity on T2-weighted spectral presaturation with inversion recovery sequence. (C) Contrast-enhanced fat-suppressed T1-weighted sequence demonstrates heterogeneously strong enhancement of the lesion. 


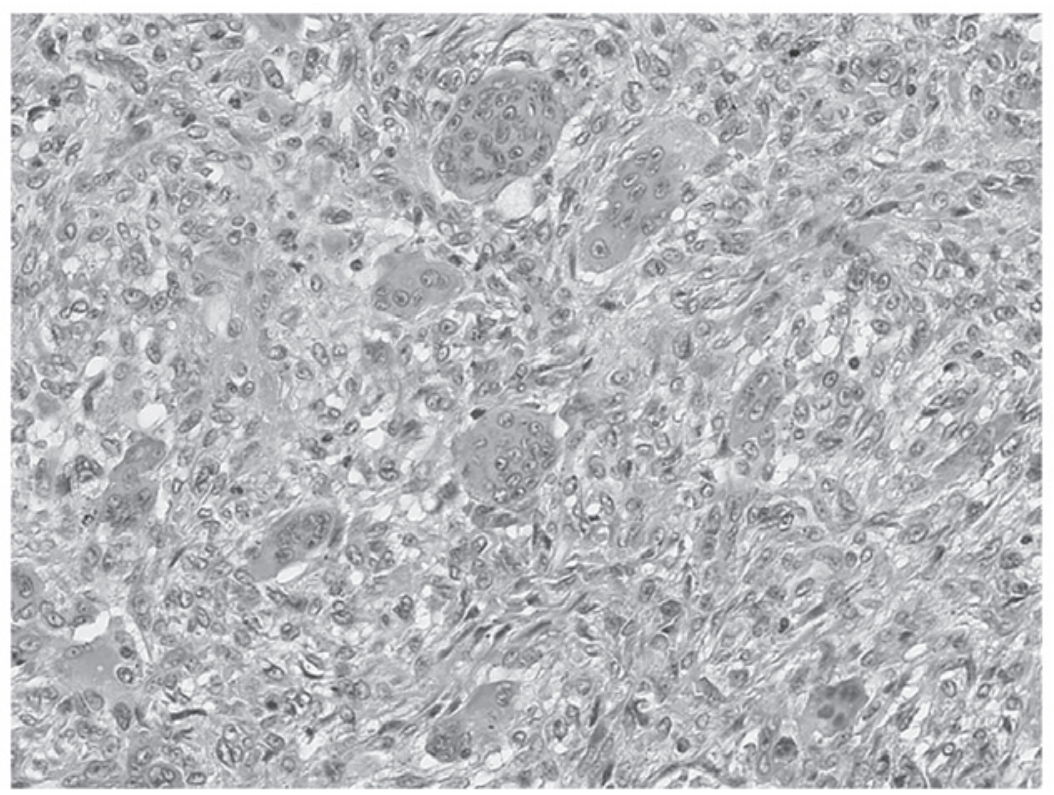

Figure 5. The tumor is composed of round or spindle-shaped mononuclear cells admixed with numerous osteoclastic giant cells.
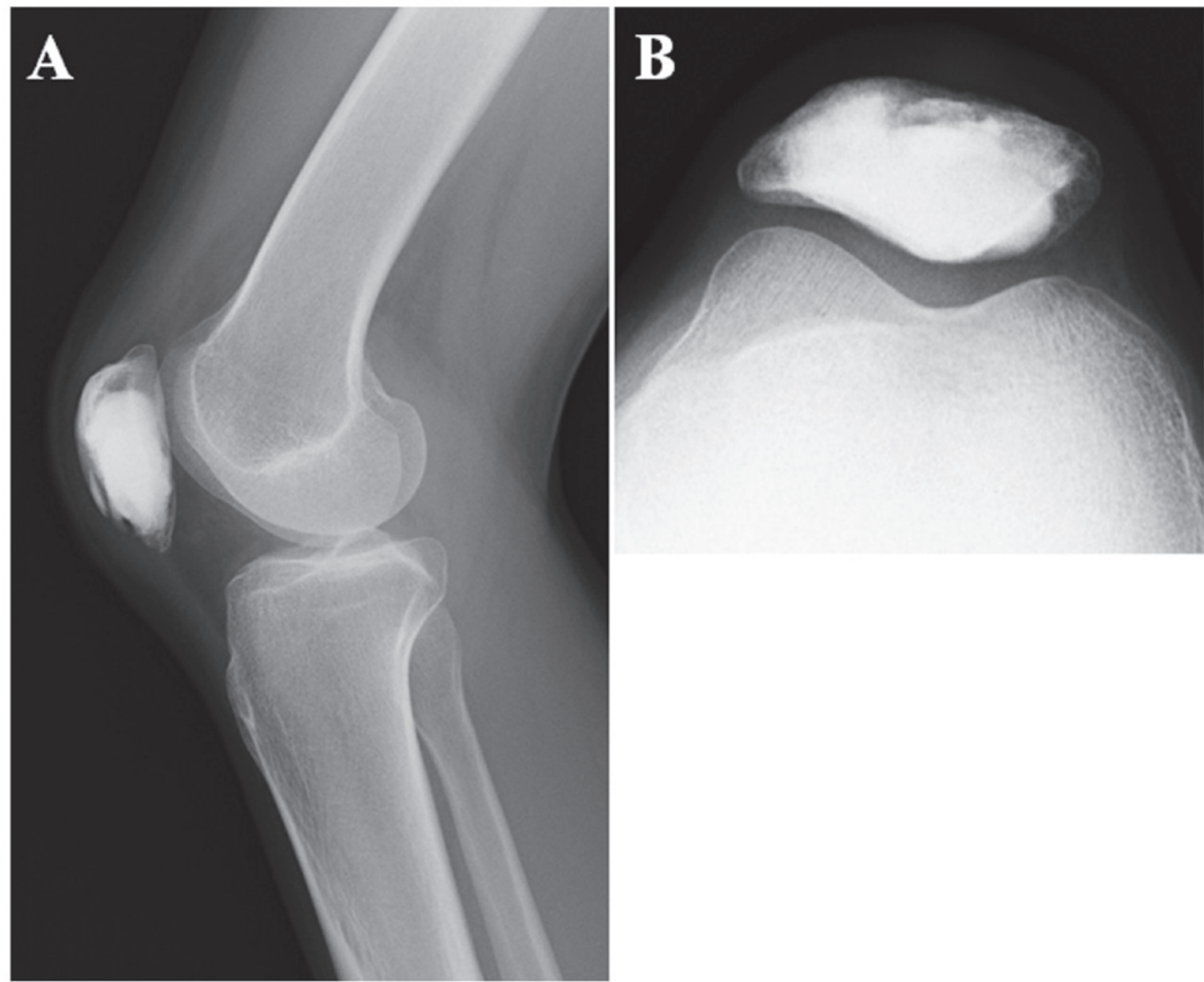

Figure 6. Follow-up (A) lateral and (B) axial radiographs of the right knee 16 months after surgery.

among the mononuclear cells, but there were no atypical mitoses identified. These findings confirmed the diagnosis of GCT of the bone.
The postoperative course was uneventful. The patient returned to normal activities within 1 month after surgery. At 16 months follow-up, the patient was asymptomatic and 
there was no evidence of local recurrence (Fig. 6) or distant metastasis.

\section{Discussion}

GCT is a benign but locally aggressive bone tumor that is composed of mononuclear cells and osteoclast-like giant cells. GCT typically affects the ends (epiphyses) of long bones, particularly the distal femur and proximal tibia. GCT has a peak incidence in the third or fourth decade of life, with a marginal female predominance (3). Pain is the most common symptom. The majority of patients note swelling of the affected region. Pathological fractures are seen in $5-10 \%$ of the patients (3). Traditionally, GCT has been treated surgically with curettage and placement of bone graft or cement. Local recurrence occurs in $15-25 \%$ of the patients (4), depending on the thoroughness of the curettage and the nature of the adjuvant therapy. Malignant transformation occurs in $<1 \%$ of all GCTs (3). The histogenesis of GCT has not been fully elucidated.

Patellar tumors represent an uncommon etiology of anterior knee pain and their diagnosis is often delayed (5). Benign lesions are more frequent compared to malignant tumors $(1,2,6)$. The most common benign tumor is GCT, followed by chondroblastoma and ABC (2). Metastases are the most frequent malignant tumors of the patella and primary malignant lesions predominantly include osteosarcoma, hemangioendothelioma and lymphoma (2). We previously reported a case of osteosarcoma of the patella mimicking GCT with secondary $\mathrm{ABC}$ in a middle-aged patient (7).

The differential diagnosis of the present case may include chondroblastoma and hemangioma. Chondroblastoma accounts for $<1 \%$ of all bone tumors and most frequently occurs in the second decade of life, with a male predominance (8). Radiographically, chondroblastoma usually presents as a well-defined, round or oval lytic lesion. Periosteal reaction is absent in the patella. Calcification is observed in $19 \%$ of the cases (2), which is less compared to other skeletal sites. CT may be useful in revealing tiny, scattered calcifications. Peritumoral edema may be seen on MRI. Chondroblastoma is usually smaller in size compared to GCT (1). On the other hand, 10 cases of hemangioma in the patella have been reported to date, representing $2 \%$ of all patellar tumors (2). Periosteal reaction, calcification and septation are absent in the patella. On MRI, bone hemangioma typically exhibits increased signal intensity on T1- and T2-weighted sequences, possibly due to the fat content of the lesion (9). In the present case, it was difficult to distinguish these 2 conditions from GCT on the basis of imaging characteristics alone.

The surgical treatment for patellar GCT includes curettage with bone graft and patellectomy $(6,10,11)$. Total patellectomy has been recommended as the preferred treatment for aggressive benign lesions with cortical breakthrough (6). Recently, Malhotra et al (12) reported a case of aggressive GCT of the patella and its management with wide resection and reconstruction of the extensor mechanism using a patellar allograft. We performed radical curettage with adjuvant phenol and ethanol and injectable calcium phosphate cement grafting. This treatment was considered to be appropriate for our patient. Sixteen months after surgery, the patient was free of symptoms and there was no clinical or radiographic evidence of local recurrence.

In summary, we have described a rare case of patellar GCT in a young adult patient. Patellar tumors, such as GCT, should be considered in the differential diagnosis of persistent unexplained knee pain, particularly in young adult patients.

\section{References}

1. Singh J, James SL, Kroon HM, Woertler K, Anderson SE, Jundt $\mathrm{G}$ and Davies AM: Tumour and tumour-like lesions of the patella - a multicentre experience. Eur Radiol 19: 701-712, 2009.

2. Casadei R, Kreshak J, Rinaldi R, Rimondi E, Bianchi G, Alberghini M, Ruggieri P and Vanel D: Imaging tumors of the patella. Eur J Radiol 82: 2140-2148, 2013.

3. Athanasou NA, Bansal M, Forsyth R, Reid RP and Sapi Z: Giant cell tumor of bone. In: World Health Organization Classification of Tumours of Soft Tissue and Bone. Fletcher CDM, Bridge JA, Hogendoorn PCW and Mertens F (eds). 4th edition. IARC Press, Lyon, pp321-324, 2013.

4. Chakarun CJ, Forrester DM, Gottsegen CJ, Patel DB, White EA and Matcuk GR Jr: Giant cell tumor of bone: review, mimics, and new developments in treatment. Radiographics 33: 197-211, 2013.

5. Saglik Y, Yildiz Y, Basarir K, Tezen E and Guner D: Tumours and tumour-like lesions of the patella: a report of eight cases. Acta Orthop Belg 74: 391-396, 2008.

6. Mercuri M and Casadei R: Patellar tumors. Clin Orthop Relat Res 389: 35-46, 2001.

7. Aoki M, Nishio J, Iwasaki H, Masaki M, Kawakami Y, Nishino T, Ohjimi H, Tamura K, Nabeshima K and Naito M: Osteosarcoma of the patella mimicking giant cell tumor: imaging features with histopathological correlation. Anticancer Res 34: 2541-2545, 2014.

8. Kilpatrick SE and Romeo S: Chondroblastoma. In: World Health Organization Classification of Tumours of Soft Tissue and Bone. Fletcher CDM, Bridge JA, Hogendoorn PCW and Mertens F (eds). 4th edition. IARC Press, Lyon, pp262-263, 2013.

9. Vilanova JC, Barcelo J, Smirniotopoulos JG, Perez-Andres R, Villalon M, Miro J, Martin F, Capellades J and Ros PR: Hemangioma from head to toe: MR imaging with pathologic correlation. Radiographics 24: 367-385, 2004.

10. Ferguson PC, Griffin AM and Bell RS: Primary patellar tumors. Clin Orthop Relat Res 336: 199-204, 1997.

11. Yoshida Y, Kojima T, Taniguchi M, Osaka S and Tokuhashi Y: Giant-cell tumor of the patella. Acta Med Okayama 66: 73-76, 2012.

12. Malhotra R, Sharma L, Kumar V and Nataraj AR: Giant cell tumor of the patella and its management using a patella, patellar tendon, and tibial tubercle allograft. Knee Surg Sports Traumatol Arthrosc 18: 167-169, 2010. 